\title{
Determination of the glass-forming concentration range in binary alloys from a semiempirical theory: Application to $\mathrm{Zr}$-based alloys
}

\author{
J. M. López* \\ School of Mathematics and Physics, University of East Anglia, Norwich NR4 7TJ, United Kingdom \\ J. A. Alonso \\ Departamento de Física Teórica, Universidad de Valladolid, Spain \\ L. J. Gallego ${ }^{\dagger}$ \\ School of Mathematics and Physics, University of East Anglia, Norwich NR4 7TJ, United Kingdom
}

(Received 2 February 1987)

\begin{abstract}
A semiempirical theory is presented to calculate free-energy diagrams of glass-forming alloys. The theory is based on the enthalpy of formation of the solid solution expressed as a sum of two contributions: (a) a chemical contribution due to electronic redistribution in forming the alloy, and (b) an elastic contribution arising from the difference in size between solute and solvent atoms. The enthalpy of formation of the amorphous phase has only the chemical contribution. The theory is used to successfully describe the glass-forming concentration range of some $\mathrm{Zr}$-based alloys, which have been experimentally analyzed by several distinct techniques.
\end{abstract}

\section{INTRODUCTION}

Growing efforts have been devoted to the promotion of a better understanding of the glass-formation ability (GFA) of metallic alloys (see, e.g., the recent review by Jackle $^{1}$ and references therein). At the same time, new powerful techniques, as the ion-beam mixing ${ }^{2-4}$ and the laser quenching, ${ }^{5-8}$ have been developed to produce amorphous materials which have not been obtained by the traditional quenching methods. Because the explanation of the amorphization mechanism on the atomic scale is a very difficult task, several semiempirical treatments have been proposed focusing on the fundamental factors which are believed to influence the GFA. For instance, Egami and co-workers 9,10 and Liou and Chien, ${ }^{11}$ by examining the published reports on the glass formation range of a large number of binary systems, have found that the minimum solute concentration necessary to obtain amorphous alloys by rapid quenching is inversely related to the atomic-volume mismatch. On the other hand, Liu ${ }^{12}$ has recently formulated rules for predicting glass formation of alloys prepared by ion beam mixing on the basis of thermodynamic and kinetic considerations. He states that (i) if the overall composition is in a two-phase region of the phase diagram, an amorphous alloy is most likely to be formed, and (ii) if the overall composition is in or near a single-phase region of the phase diagram and the structure of this phase is not simple, an amorphous alloy is likely to be formed. The first of these rules is called the "extendedstructural-difference rule," and is more general than the well-known deep eutectic criterion for forming metallic glasses by rapid liquid melt quenching. Both of the rules proposed by Liu are consistent with the idea that only chemically different metals are able to form amorphous alloys.
Another approach to the question of the GFA has been developed by Alonso and co-workers ${ }^{13-16}$ using a two-dimensional map formed by thermochemical coordinates. In a recent paper published by these authors, ${ }^{15}$ they use the heat of mixing in the liquid state and the size-mismatch contribution to the heat of formation of the solid solution, calculated from elasticity theory, to construct glass-forming maps. These maps are able to perfectly separate amorphous and nonamorphous alloys which have been treated by ion beam mixing and laserquenching techniques. Thermochemical coordinates were also successfully employed by the authors to predict solid solubility in equilibrium alloys ${ }^{17-19}$ and to differentiate between substitutional and interstitial solute positions in dilute alloys obtained by ion implantation. ${ }^{20,21}$

The main virtue of the thermochemical-coordinate approach is that it provides a route to quantitative calculations of the free energy of mixing of alloy phases, and then to construct equilibrium or metastable phase diagrams. This has been successfully done for the case of equilibrium solid solutions, ${ }^{22}$ and we now propose to consider the metastable case. As we shall show, our treatment allows a very accurate prediction of the glass forming concentration range of several metallic systems, which emphasizes the role of the thermochemical coordinates in controlling the formation of amorphous alloys.

The layout of this paper is as follows. In Sec. II we briefly survey the basic thermodynamic relations on which the construction of the free-energy diagrams carried out in this paper is based. In Sec. III we present the semiempirical theory which allows us to obtain the enthalpies of mixing of the competing phases existing in alloys with GFA. In Sec. IV we apply the theory to study the glass forming concentration range of some $\mathrm{Zr}$ based alloys, which have been experimentally studied by 
several distinct techniques (see Saunders and Miodownik ${ }^{23}$ and references therein). Finally, the conclusions are presented in Sec. V. The good results obtained in this paper provide, we think, a suitable test of the reliability of the approximations used.

\section{THERMODYNAMIC RELATIONS}

The glass forming range of a binary alloy $A-B$ is limited by the composition at which the Gibbs free energies of competing crystalline phases become more negative than that of the amorphous phase, and the possible presence of structurally simple compounds (see Liu ${ }^{12}$ and discussion below). As usual, we shall treat the amorphous phase as an extension of the liquid phase to temperatures in the undercooled regime, thus neglecting the configurational freezing of the undercooled liquid as the glass transition temperature, $T_{g}$, is approached from above. The construction of the free energy diagrams will be carried here by using the free energies of mixing, rather than the Gibbs free energies as is usually done (e.g., Schwarz and co-workers ${ }^{24-26}$ ). by

The free energy of mixing of a solid solution is given

$$
\Delta G_{S}=G_{S}-X_{A} G_{A, S}-X_{B} G_{B, S},
$$

where $G_{A, S}$ and $G_{B, S}$ are the free energies of the pure solid metals $A$ and $B$, and $G_{S}$ is the free energy of the solid solution with atomic concentrations $X_{A}$ and $X_{B}$. $\Delta G_{S}$ can be separated as

$$
\Delta G_{S}=\Delta H_{S}-T \Delta S_{S},
$$

where $\Delta H_{S}$ and $\Delta S_{S}$ are the enthalpy and entropy of mixing of the crystalline phase, respectively. To determine the glass forming concentration range of the alloys, $\Delta G_{S}$ must be compared with the excess of the free energy of the amorphous or undercooled melt over those of the pure solid metals, i.e., with the quantity

$$
\overline{\Delta G}_{l} \equiv G_{l}-X_{A} G_{A, S}-X_{B} G_{B, S},
$$

where $G_{l}$ is the free energy of the amorphous phase. $\overline{\Delta G}_{l}$ can also be written as

$$
\overline{\Delta G}_{l}=\overline{\Delta H}_{l}-T \overline{\Delta S}_{l},
$$

where $\overline{\Delta H}_{l}$ and $\overline{\Delta S}_{l}$ are defined, respectively, by

$$
\overline{\Delta H}_{l}=H_{l}-X_{A} H_{A, S}-X_{B} H_{B, S}
$$

and

$$
\overline{\Delta S}_{l}=S_{l}-X_{A} S_{A, S}-X_{B} S_{B, S} .
$$

In these expressions $H_{l}$ and $S_{l}$ are the enthalpy and entropy of the amorphous phase, respectively, and the quantities $H_{A, S}, H_{B, S}, S_{A, S}$, and $S_{B, S}$ refer to the pure solid components $A$ and $B . \overline{\Delta G}_{l}$ can be related to the true free energy of mixing of the amorphous phase, $\Delta G_{l}$, as indicated immediately below.

We first note that, at a given temperature, the enthalpies of the solid and the undercooled pure metals are related through the so-called Kirchhoff's equation
$H_{i, l}-H_{i, S}=\Delta H_{f, i}-\int_{T}^{T_{f, i}}\left(C_{p, i}^{l}-C_{p, i}^{S}\right) d T \quad(i=A, B)$

where $\Delta H_{f, i}$ is the heat of fusion of the component $i$, $T_{f_{S} i}$ is the melting point of this component, and $C_{p, i}^{l}$ and $C_{p, i}^{S}$ are the specific heats, at constant pressure, of the component $i$ in the undercooled and in the solid state, respectively. In a similar way, one can write for the entropies the relation

$S_{i, l}-S_{i, S}=\frac{\Delta H_{f, i}}{T_{f, i}}-\int_{T}^{T_{f, i}}\left(C_{p, i}^{l}-C_{p, i}^{S}\right) \frac{d T}{T} \quad(i=A, B)$.

To calculate the integral terms in Eqs. (7) and (8) several approximations can be used (see Ref. 27). The simplest approximation, first proposed by Turnbull, ${ }^{28}$ assumes that the difference $\Delta C_{p, i}=C_{p, i}^{l}-C_{p, i}^{S}$ is zero, which leads to

$$
H_{i, l}-H_{i, S}=\Delta H_{f, i} \quad(i=A, B)
$$

and

$$
S_{i, l}-S_{i, S}=\frac{\Delta H_{f, i}}{T_{f, i}} \quad(i=A, B) .
$$

This approximation has been successfully employed by Schwarz et al. ${ }^{24,25}$ to study the formation of amorphous alloys by solid-state reaction. Several improvements on Eqs. (9) and (10) have been published. ${ }^{29-31}$ Perepezko and Paik $^{27}$ have analyzed these approximations in terms of measurements of $\Delta C_{p}$ in undercooled melts of $\mathrm{Hg}, \mathrm{In}$, $\mathrm{Sn}$, and $\mathrm{Bi}$. Their conclusion is that a linear variation of $\Delta C_{p}$ with $T$, as proposed by Singh and Holtz, ${ }^{31}$ gives the best agreement with the experimental data. In spite of this result, it must be said that the use of better approximations than that provided by Eqs. (9) and (10) has rather little effect on the products and the composition regimes predicted by the free energy diagram, as pointed out by Schwarz et al. ${ }^{25}$ Accordingly, we have kept these equations in the calculations carried out in this paper. Then, using the expressions (4)-(6), (9), and (10), one finds that the quantities $\overline{\Delta G}_{l}$ and $\Delta G_{l}$ are related by

$$
\begin{aligned}
\overline{\Delta G}_{l}= & \Delta G_{l}+X_{A} \frac{\Delta H_{f, A}\left(T_{f, A}-T\right)}{T_{f, A}} \\
& +X_{B} \frac{\Delta H_{f, B}\left(T_{f, B}-T\right)}{T_{f, B}},
\end{aligned}
$$

where $\Delta G_{l}$, the free energy of mixing of the amorphous phase, is given by

$$
\begin{aligned}
\Delta G_{l}= & \left(H_{l}-X_{A} H_{A, l}-X_{B} H_{B, l}\right) \\
& -T\left(S_{l}-X_{A} S_{A, l}-X_{B} S_{B, l}\right) \\
= & \Delta H_{l}-T \Delta S_{l} .
\end{aligned}
$$

In the calculations performed in this paper with Eqs. (2), (11), and (12), we have assumed that the entropy of mixing, of both solid solutions and amorphous phases, is 
that corresponding to an ideal mixing, i.e.,

$$
\Delta S=-R\left(X_{A} \ln X_{A}+X_{B} \ln X_{B}\right),
$$

where $R$ is the gas constant. Of course, better approximations for the entropy of mixing can be used, but for our purposes ideality leads to good results. The next step is to set up expressions for the enthalpies of mixing of the competing phases. This point is considered in the next section.

\section{ENTHALPY OF FORMATION OF METALLIC SOLID AND AMORPHOUS SOLUTIONS}

The enthalpy of mixing, or enthalpy of formation, of a substitutional solid solution contains three terms: ${ }^{22,32,33}$

$$
\Delta H_{S}=\Delta H_{S}^{c}+\Delta H_{S}^{\text {size }}+\Delta H_{S}^{\text {str }} .
$$

The first one, $\Delta H_{S}^{c}$, is a chemical term due to the electron redistribution that occurs when the alloy is formed. It has been extensively studied by Miedema and coworkers. $^{34-36}$ According to these authors, $\Delta H_{S}^{c}$ can be written as

$$
\Delta H_{S}^{c}=\Delta H^{\mathrm{amp}} X_{A} V_{A}^{2 / 3} f_{A B},
$$

where $\Delta H^{\text {amp }}$ is an amplitude concerning the magnitude of the chemical interaction (see, for instance, Ref. 34 for details), $V_{A}$ is the atomic volume of metal $A$ in the alloy, and $f_{A B}$ is a function which accounts for the degree to which atoms of type $A$ are surrounded by atoms of type $B$. For statistical solutions $f_{A B}$ is given by

$$
f_{A B}=X_{B}^{S},
$$

where $X_{B}^{S}$ is the atomic cell surface-area concentration of metal $B$ in the alloy. For solid ordered compounds Miedema and co-workers use the empirical relation

$$
f_{A B}=X_{B}^{S}\left[1+\gamma\left(X_{A}^{S} X_{B}^{S}\right)^{2}\right]
$$

with $\gamma=8$

The enthalpy of formation of an amorphous alloy has sometimes been calculated using Eqs. (15) and (16) and then adding a constant to account for the enthalphy difference between a crystalline and an amorphous element (see, e.g., Ref. 37). Weeber ${ }^{38}$ has modified the function $f_{A B}$, this modification being based on the existence of chemical short-range order (CSRO) in amorphous alloys. In this way, Weeber suggested to use (17) with $\gamma=5$.

$\Delta H_{S}^{\text {size }}$ is an elastic contribution due to the difference in atomic volume between solute and solvent metals. This term can be computed using classical elasticity theory. ${ }^{33,39,40}$ The result can be written in the following simple form ${ }^{22,33}$

$$
\Delta H_{S}^{\text {size }}=\frac{X_{A} X_{B} \Delta h_{S}^{\text {size }}(A \text { in } B) \Delta h_{S}^{\text {size }}(B \text { in } A)}{X_{A} \Delta h_{S}^{\text {size }}(A \text { in } B)+X_{B} \Delta h_{S}^{\text {size }}(B \text { in } A)},
$$

where $\Delta h_{S}^{\text {size }}(A$ in $B)$ is the size mismatch contribution to the enthalpy of solution of $A$ in $B$, and their expression can be found, for instance, in Ref. 22.
The size mismatch contribution is not important in intermetallic compounds or in amorphous alloys. In the first case it is because, despite the size difference between the $A$ and $B$ atomic volumes, the atoms always find the proper crystal structure and concentration in order to avoid size mismatch contributions to the enthalpy of formation (the size mismatch contribution is always positive). In the amorphous phase this contribution is not important because there is not a periodic structure, and so the atoms are not constrained to fit a certain volume.

Finally $\Delta H_{S}^{\text {str }}$ of Eq. (14) is a structural contribution taking into account the difference in valence and crystal structure of solute and solvent. ${ }^{22,32}$ This contribution is expected to have only a minor effect in determining the concentration range of the amorphous alloys treated here. This is because the size effect, which is the primary factor controlling this concentration range (as Egami $^{9,10}$ and Liou ${ }^{11}$ have emphasized), is very strong in the alloys treated in this paper. For this reason $\Delta H_{S}^{\text {str }}$ will not be considered. In addition there are technical reasons which make difficult the handling of $\Delta H_{S}^{\text {str }}$. These will be briefly discussed in Sec. IV.

All the above results allow us to obtain the free energies of the amorphous and solid phases of metallic systems. This is illustrated in the next section by considering some Zr-based alloys whose amorphization has been studied by several distinct techniques.

\section{CALCULATIONS FOR Zr-BASED ALLOYS AND DISCUSSION}

We have calculated the free energy of formation of the amorphous and the solid phases, as a function of concentration, for the systems $\mathrm{Ni}-\mathrm{Zr}, \mathrm{Co}-\mathrm{Zr}$, and $\mathrm{Cu}-\mathrm{Zr}$. To obtain $\overline{\Delta G}_{l}$ we have calculated $\Delta G_{l}$ by using Eq. (12) with $\Delta S_{l}$ and $\Delta H_{l}$ given by (13) and (15), respectively. Moreover, the second and third terms on the right-hand side of Eq. (11) have been evaluated by using the experimental melting points and heat of fusion data. ${ }^{41}$ The expression used for the function $f_{A B}$ of Eq. (15) in the amorphous phase is that proposed by Weeber, ${ }^{38}$ that is Eq. (17), but with $\gamma=4$. We have obtained this optimized value of $\gamma$ by fitting the available $\overline{\Delta H}_{l}$ data for the amorphous systems $\mathrm{Ni}-\mathrm{Zr}$ (Refs. 23 and 37) and $\mathrm{Cu}-\mathrm{Zr}$ (Refs. 42 and 43) to the expression

$$
\overline{\Delta H}_{l}=\Delta H_{l}+X_{A} \Delta H_{f, A}+X_{B} \Delta H_{f, B},
$$

which results from Eqs. (5) and (9). As an illustration in Fig. 1 we have plotted the $\overline{\Delta H}_{l}$ fit corresponding to the system Ni-Zr.

On the other hand we have obtained the free energy of mixing of the solid solution by using Eqs. (15) and (18) to obtain the enthalpy of mixing, with $\gamma=4$ in the expression (17) for $f_{A B}$, and Eq. (13) for the entropy of mixing. Using $\gamma=4$ in $f_{A B}$ means that we admit the existence of CSRO in the solid solution (suggested by the very negative heat of formation of the alloys considered in this paper) and that the degree of CSRO is similar to that in the corresponding amorphous phase. This of course implies that $\Delta H_{l}=\Delta H_{S}^{c}$.

The results of the free energy calculations for the sys- 
tems $\mathrm{Ni}-\mathrm{Zr}, \mathrm{Co}-\mathrm{Zr}$, and $\mathrm{Cu}-\mathrm{Zr}$ at $300 \mathrm{~K}$ are plotted in Figs. 2-4. In these figures the theoretical range of amorphization has been obtained by considering the solid solution and the amorphous phase as the only competitive phases. Thus, when the concentration of $\mathrm{Zr}$ is smaller than that corresponding to the point indicated in the figures with the number 1, or greater than the concentration corresponding to the point indicated by 4 , the more stable phase is the solid solution and the system is not amorphizable. When the concentration is between the values corresponding to the points 1 and 4 , the free energy of the amorphous alloy is more negative than the free energy of solid solutions and the system becomes amorphous.

So far we have not considered the existence of intermetallic compounds in the phase diagram. Generally these compounds have a complicated crystal structure and thus they are not usually competing phases due to nucleation and growth difficulties under rapid quenching conditions. This is corroborated by the studies of the thermal stability in amorphous alloys carried out by Buschow. $^{44}$ According to him, the regions with minimum stability, i.e., with a minimum in the activa-

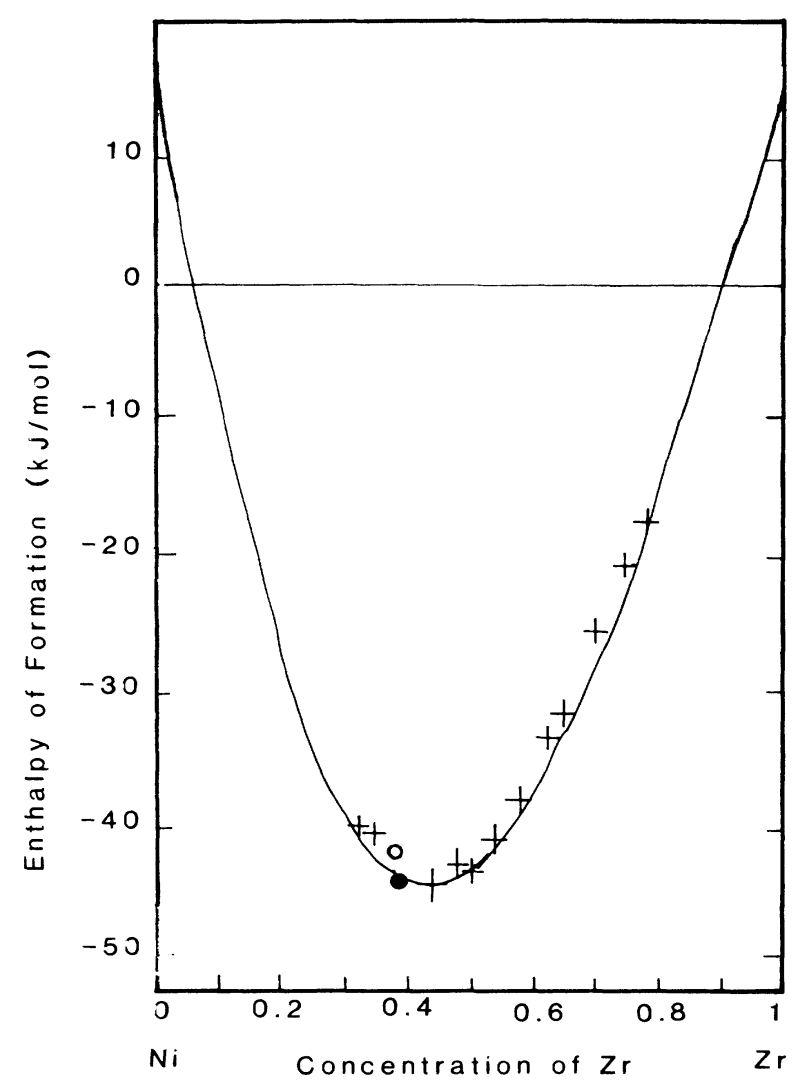

FIG. 1. Enthalpy of formation, $\overline{\Delta H}_{l}$, for the amorphous $\mathrm{Ni}-\mathrm{Zr}$ system vs concentration of $\mathrm{Zr}$ at $300 \mathrm{~K}$. The solid line is the theoretical fit given by Eq. (19) to the experimental data (crosses; Refs. 23 and 37). We have also superimposed on this figure the values reported by Altounian et al. (open circle; Ref. 50) and by Weeber et al. (solid circle; Ref. 51). tion energy for crystallization, correspond to concentrations at which intermetallic compounds occur. This fact indicates that the possible formation of amorphous phases at these concentrations is due to kinetic frustration of the crystallization process (see Ref. 12). In Figs. 2-4 the intermetallic compounds are indicated by arrows at the nominal concentrations. For the $\mathrm{Ni}-\mathrm{Zr}$ system there are seven intermediate compounds ${ }^{45}$ which have complicated structures, with the exception of $\mathrm{Ni}_{5} \mathrm{Zr}$ which is of the fcc type. This is the same structure as that of the solid solution in the Ni-rich concentration. The Co- $\mathrm{Zr}$ system has five intermetallic compounds; ${ }^{45}$ only the $\mathrm{Co}_{2} \mathrm{Zr}$ is fcc type. Finally, the $\mathrm{Cu}-\mathrm{Zr}$ system has ten intermediate compounds, ${ }^{46}$ nine of which have a complicated structure and only one, the $\mathrm{Cu}_{5} \mathrm{Zr}$, is fcc type, as pure $\mathrm{Cu}$.

In these three systems studied we have calculated the free energy of formation for the compound that has the same type of crystallographic structure as the corre-

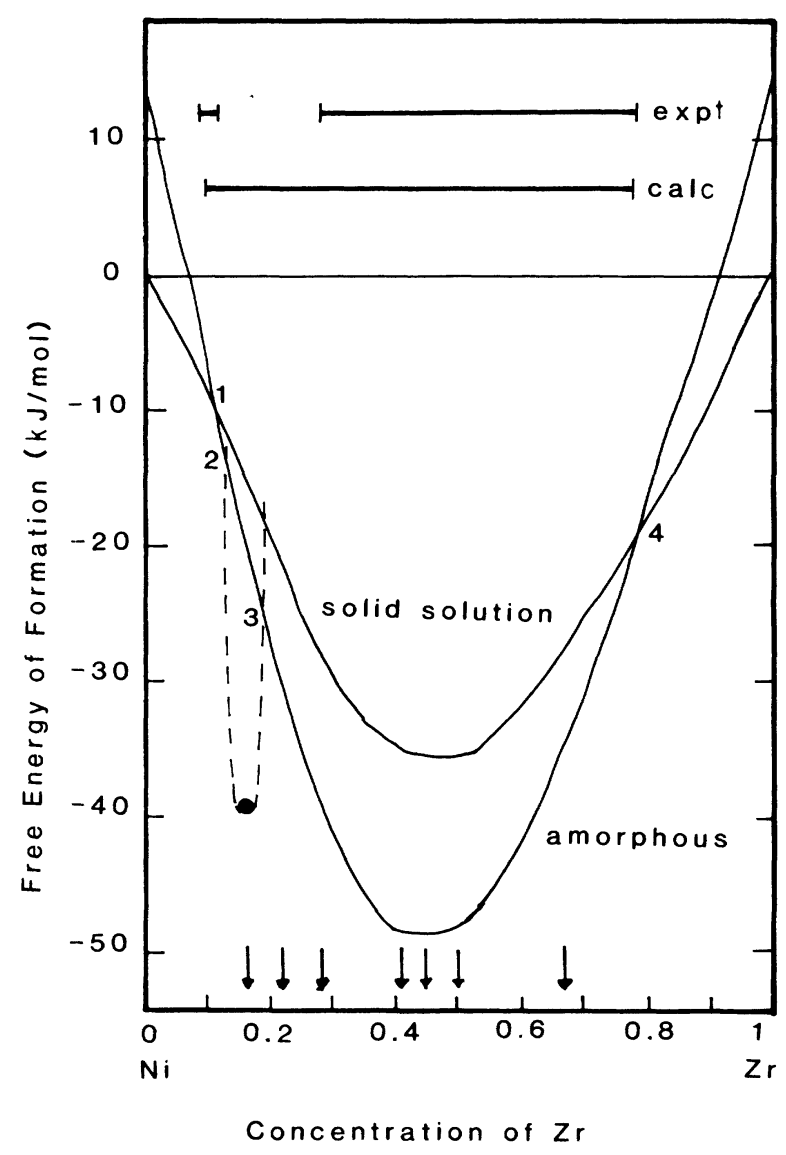

FIG. 2. Calculated free energies of formation, $\overline{\Delta G}_{l}$ and $\Delta G_{S}$, for the $\mathrm{Ni}-\mathrm{Zr}$ system at $300 \mathrm{~K}$ vs concentration of $\mathrm{Zr}$. The solid circle is the free energy of formation of the $\mathrm{Ni}_{5} \mathrm{Zr}$ compound. The dashed line is schematic. In the upper part of the figure we show the experimentally observed glass-forming range found on melt spinning (Ref. 44) and that calculated in this work. Arrows indicate positions of equilibrium compounds. 
sponding terminal solid solution. We have assumed here that the structure of the pure Co is fcc type. However, it must be said that this metal has two crystallographic forms, one of hcp type ( $\alpha-\mathrm{Co})$ and the other of fcc type $(\beta-\mathrm{Co})$, both forms commonly coexisting in samples at room temperature (see, e.g., Ref. 47). The free energy calculations have been carried out using Eq. (15) for the enthalpy of formation, with $f_{A B}$ given by (17) $(\gamma=8)$, and assuming $\Delta S_{\text {compound }}=0$, because evidence exists that the entropy of formation is smaller than the entropy of formations of the solid solutions. ${ }^{48,49}$ The results are indicated by a filled circle in Figs. 2-4; a schematic curve (dashed line) has been drawn to indicate that the free energy of the intermetallic compound changes rapidly around the nominal composition. This allows us to explain the existence of a gap in the experimental range of amorphization for the three systems considered in this

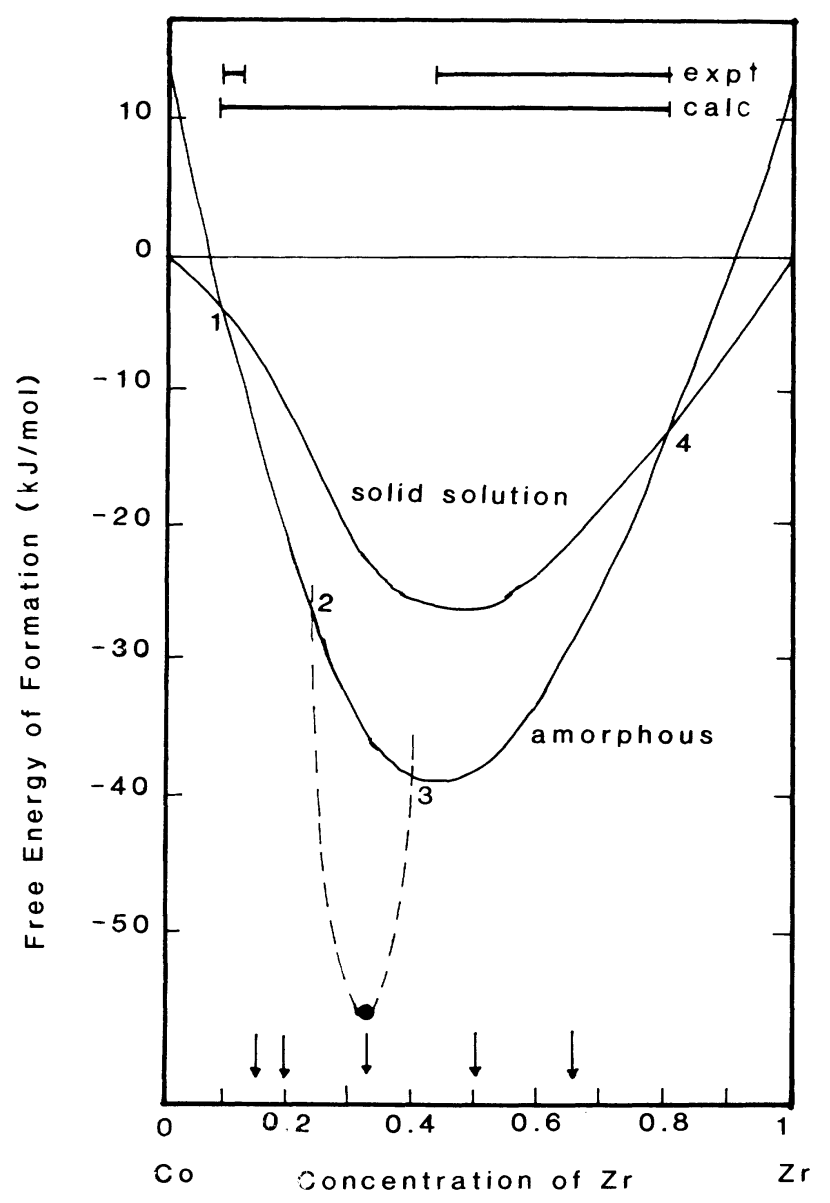

FIG. 3. Calculated free energies of formation, $\overline{\Delta G}_{l}$ and $\Delta G_{S}$, for the Co- $\mathrm{Zr}$ system at $300 \mathrm{~K}$ vs concentration of $\mathrm{Zr}$. The filled circle is the free energy of formation of the $\mathrm{Co}_{2} \mathrm{Zr}$ compound. The dashed line is schematic. In the upper part of the figure we show the experimentally observed glass-forming range found on melt spinning (Ref. 44) and that calculated in this work. Arrows indicate positions of equilibrium compounds. paper. When the concentration of the system is between those points indicated in the figures by numbers 2 and 3, the simpler and more stable phase is in the intermetallic compound. Thus, when the system is rapidly cooled from the liquid state this is the phase that is obtained rather than the amorphous phase. We can consider the stable compound basically as an extension of the solid solutions because in all cases the crystallographic structures are the same in both phases.

The good agreement with the experimental concentration range for amorphization shows that the structural contribution in Eq. (14) can be neglected, as expected, in studying this particular property. On the other hand there are technical reasons that make the handling of $\Delta H_{S}^{\text {str }}$ difficult, at least for the class of alloys treated here. The main problem is that a study of the variation of the structural stability of elemental metals as a function of the number of valence electrons has only been performed, so far, for transition metals of the $4 d$ and $5 d$ periods, ${ }^{32}$ whereas two alloys treated here contain one $3 d$ metal $(\mathrm{Co}, \mathrm{Ni})$ and the third one a noble metal $(\mathrm{Cu})$. Due to magnetic effects in the $3 d$ period, there is no guarantee at all that the data for $4 d$ and $5 d$ metals could be extrapolated with confidence to the $3 d$ period. In fact $\mathrm{Mn}, \mathrm{Fe}$, and Co have crystal structures different from those of their corresponding $4 d$ or $5 d$ partners. Even if this extrapolation were valid, a second possible problem

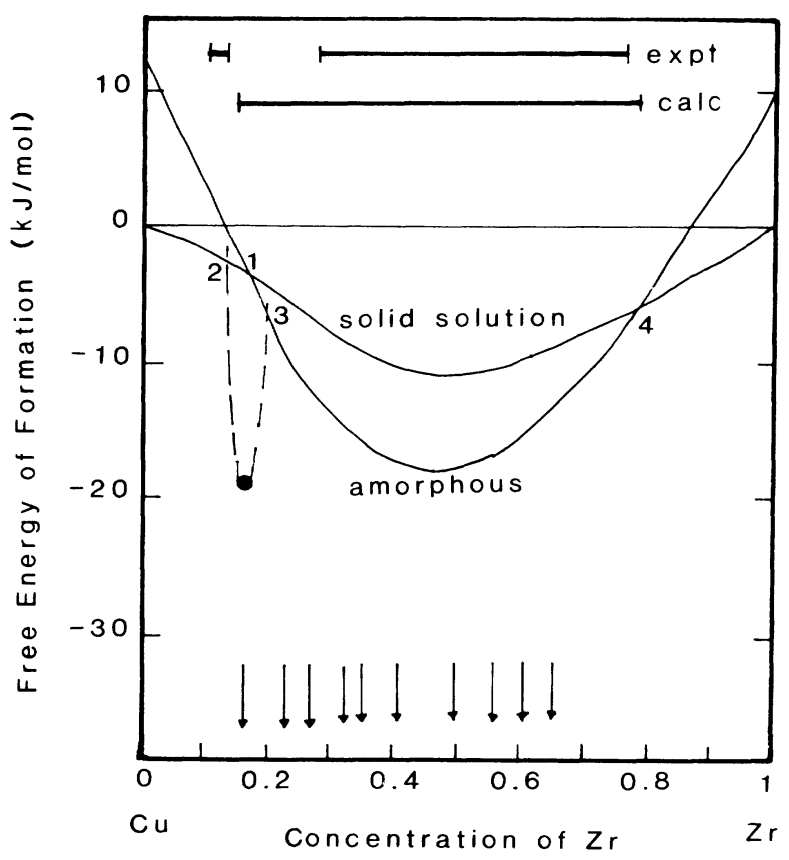

FIG. 4. Calculated free energies of formation, $\overline{\Delta G}_{l}$ and $\Delta G_{S}$, for the $\mathrm{Cu}-\mathrm{Zr}$ system at $300 \mathrm{~K}$ vs concentration of $\mathrm{Zr}$. The filled circle is the free energy of formation of the $\mathrm{Cu}_{5} \mathrm{Zr}$ compound. The dashed line is schematic. In the upper part of the figure we show the experimentally observed glass-forming range found on melting spinning (Ref. 44) and that calculated in this work. Arrows indicate positions of equilibrium compounds. 
could be the large valence difference between $\mathrm{Zr}$ and $\mathrm{Ni}$, $\mathrm{Co}$ or $\mathrm{Cu}$. In spite of these reservations we could adopt the questionable point of view of taking the structural stability curves of Fig. 2 in Ref. 32 as valid for the nonmagnetic $3 d$ metals. Then, after including $\Delta H_{S}^{\text {str }}$ in our calculations for the $\mathrm{Zr}-\mathrm{Ni}$ alloy we have found that the concentration range for amorphization becomes hardly modified $(\sim 1$ at. \%) with respect to the amorphization range in Fig. 2. This is, of course, because the size effect is the dominant factor.

Evidently, from the theory developed above, the crossing points between the curves $\overline{\Delta G}_{l}$ and $\Delta G_{S}$ in Figs. 2-4 are determined by the equation

$$
\begin{aligned}
X_{A} \frac{\Delta H_{f, A}\left(T_{f, A}-T\right)}{T_{f, A}}+X_{B} \frac{\Delta H_{f, B}\left(T_{f, B}-T\right)}{T_{f, B}} \\
=\frac{X_{A} X_{B} \Delta h_{S}^{\operatorname{size}}(A \text { in } B) \Delta h_{S}^{\operatorname{size}}(B \text { in } A)}{X_{A} \Delta h_{S}^{\operatorname{size}}(A \text { in } B)+X_{B} \Delta h_{S}^{\operatorname{size}}(B \text { in } A)} .
\end{aligned}
$$

This result agrees with the findings of Egami and coworkers $^{9,10}$ and of Liou and Chien ${ }^{11}$ that atomic size mismatch between the components is critical in determining the concentration range for amorphization. Rather than working directly with Eq. (20) we have preferred, however, to plot in the figures the free energy of formation of the competing phases because this gives a more comprehensive view of the situation, and it also allows for a simpler discussion of the effect of intermediate compounds.

\section{SUMMARY}

In conclusion, we have presented a theory which is capable of successfully explaining the concentration range in which amorphous alloys can be obtained in binary transition-metal systems. Although this theory is semiempirical we believe that it contains the fundamental thermodynamic factors influencing glass forming ability. The theory emphasizes the role of the size mismatch contribution in determining the glass-forming concentration range, whose importance has also been noted by Egami and Waseda ${ }^{9}$ and by Liou and Chien. ${ }^{11}$ The theory also takes into account the chemical contributions due to the electronic redistribution in forming the alloy. It is these factors that we believe are the key ingredients in describing the formation of amorphous alloys correctly and which any ab initio theory should incorporate. Although size mismatch effects control the glass forming concentration range, provided that the amorphous alloy forms, the chemical interaction term is important, in addition to the size term, to determine if an amorphous phase will form at all. ${ }^{13-16}$

\section{ACKNOWLEDGMENTS}

We acknowledge support by the Comisión Asesora de Investigación Cientifica y Técnica of Spain (Grant No. 3265-83) and also a grant from the Anglo/Spanish Joint Research Program (Acciones Integradas; Project 35/34). Two of us are grateful for additional support from NATO (J.M.L.) and from the British Council and the Spanish Ministerio de Educación y Ciencia (L.J.G.), enabling us to visit the University of East Anglia, where part of this work was done. The authors are grateful to M. Silbert for useful comments on this paper.
*Permanent address; Departamento de Física Teórica, Universidad de Valladolid, Spain.

†Permanent address: Departamento de Física de la Materia Condensada, Universidad de Santiago de Compostela, Spain.

${ }^{1}$ J. Jackle, Rep. Prog. Phys. 49, 171 (1986).

${ }^{2}$ W. F. van der Weg, D. Sigurd, and J. W. Mayer, in Applications of Ion Beams to Metals, edited by S. T. Picraux, N. P. Eernisse and F. L. Vook (Plenum, New York, 1974), p. 1209.

${ }^{3}$ S. Matteson and M. A. Nicolet, in Metastable Materials Formation by Ion Implantation, Vol. 7 of the Materials Research Society Symposia Proceedings, edited by S. T. Picraux and W. J. Choyke (North-Holland, Amsterdam, 1982), p.3.

${ }^{4}$ S. Matteson, IEEE Trans. Nucl. Sci. 30, 1705 (1983).

${ }^{5}$ M. von Allmen, Topics in Applied Physics, edited by H. Beck and H. J. Güntherodt (Springer, Berlin, 1983), p. 261.

${ }^{6} \mathrm{M}$. von Allmen, E. Huber, A. Blatter, and K. Affolter, Int. J. Rapid. Solidif. 1, 15 (1984-85).

${ }^{7}$ A. Blatter and M. von Allmen, Phys. Rev. Lett. 54, 2103 (1985).

${ }^{8}$ E. Huber and M. von Allmen, Phys. Rev. B 31, 3338 (1985).

${ }^{9}$ T. Egami and Y. Waseda, J. Non-Cryst. Solids 64, 113 (1984).

${ }^{10}$ T. Egami and S. Aur, J. Non-Cryst. Solids 89, 60 (1987).

${ }^{11}$ S. H. Liou and C. L. Chien, Phys. Rev. B 35, 2443 (1987).

${ }^{12}$ B. X. Liu, Phys. Status Solidi A 94, 11 (1986).
${ }^{13}$ J. A. Alonso and S. Simozar, Solid State Commun. 48, 765 (1983).

${ }^{14}$ J. A. Alonso and J. M. López, Mater. Lett. 4, 316 (1986).

15 J. A. Alonso and J. M. López, Z. Phys. Chem. (to be published).

${ }^{16}$ S. Simozar and J. A. Alonso, Phys. Status Solidi A 81, 55 (1984).

${ }^{17}$ J. A. Alonso and S. Simozar, Phys. Rev. B 22, 5583 (1980).

${ }^{18}$ J. A. Alonso, J. M. López, S. Simozar, and L. A. Girifalco, Acta Metall. 30, 105 (1982).

${ }^{19}$ J. M. López and J. A. Alonso, Physica 113B, 103 (1982).

${ }^{20}$ J. A. Alonso and J. M. López, Philos. Mag. A 45, 713 (1982).

${ }^{21}$ J. M. López and J. A. Alonso, Phys. Status Solidi A 72, 777 (1982).

22J. M. López and J. A. Alonso, Z. Naturforsch., Teil A 40, 1199 (1985).

${ }^{23}$ N. Saunders and A. P. Miodownik, J. Mater. Res. 1, 38 (1986).

${ }^{24}$ R. B. Schwarz and W. L. Johnson, Phys. Rev. Lett. 51, 415 (1983).

${ }^{25}$ R. B. Schwarz, K. L. Wong, and W. L. Johnson, J. NonCryst. Solids 61\&62, 129 (1984).

${ }^{26}$ R. B. Schwarz, R. R. Petrich, and C. K. Sawck, J. NonCryst. Solids 76, 281 (1985). 
${ }^{27}$ J. H. Perepezko and J. S. Paik, J. Non-Cryst. Solids 61\&62, 113 (1984).

${ }^{28}$ D. Turnbull, J. Chem. Phys. 20, 411 (1952).

${ }^{29}$ J. D. Hoffman, J. Chem. Phys. 29, 1192 (1958).

${ }^{30}$ C. V. Thompson and F. Spaepen, Acta Metall. 27, 1955 (1979).

${ }^{31}$ H. B. Singh and A. Holtz, Solid State Commun. 45, 985 (1983).

${ }^{32}$ A. K. Niessen and A. R. Miedema, Ber. Bunsenges. Phys. Chem. 87, 717 (1983).

${ }^{33}$ J. M. López and J. A. Alonso, Phys. Status Solidi A 76, 675 (1983); 85, 423 (1984).

${ }^{34}$ A. R. Miedema and A. K. Niessen, Comput. Coupling Phase Diagrams \& Thermochem. 7, 27 (1983).

${ }^{35}$ A. R. Miedema, F. R. de Boer, and P. F. de Châtel, J. Phys. F 3, 1558 (1973)

${ }^{36}$ A. R. Miedema, P. F. de Châtel, and F. R. de Boer, Physica B100, 1 (1980).

${ }^{37}$ M. P. Henaff, C. Colinet, A. Pasturel, and K. H. J. Buschow, J. Appl. Phys. 56, 307 (1984).

${ }^{38}$ A. W. Weeber, J. Phys. F. 17, 809 (1987).

${ }^{39}$ J. Friedel, Adv. Phys. 3, 446 (1954).

${ }^{40}$ J. D. Eshelby, Solid State Phys. 3, 79 (1956).
${ }^{41}$ K. A. Gschneidner, Jr., Solid State Phys. 16, 275 (1964).

${ }^{42}$ A. J. Kerns, D. E. Polk, R. Ray, and B. C. Giessen, Mater. Sci. Eng. 38, 49 (1979).

${ }^{43}$ I. Ansara, A. Pasturel, and K. H. Buschow, Phys. Status Solidi A 69, 447 (1982).

${ }^{44}$ K. H. J. Buschow, J. Phys. F 14, 593 (1984).

${ }^{45}$ R. P. Elliott, Constitution of Binary Alloys, First Supplement (McGraw-Hill, New York, 1965).

${ }^{46}$ E. Kneller, Y. Khan, and U. Gorres, Z. Metallkd. 77, 43 (1986).

${ }^{47}$ R. Hultgren, P. D. Desay, D. T. Hawkins, M. Gleiser, K. K. Kelley, and D. D. Wagman, Selected Values of the Thermodynamic Properties of the Elements (American Society for Metals, Metals Park, Ohio, 1973).

${ }^{48}$ B. M. Mogutnov and L. A. Shvartsman, Zh. Fiz. Khim. 54, 568 (1980) [Russ. J. Phys. Chem. 59, 328 (1980)].

${ }^{49}$ F. R. de Boer, R. Boom, and A. R. Miedema, Physica B101, 294 (1980).

${ }^{50}$ Z. Altounian, T. Gon-hua, and J. O. Strom-Olsen, J. Appl. Phys. 54, 3111 (1983).

${ }^{51}$ A. W. Weeber, H. Bakker, and F. R. de Boer, Europhys. Lett. 2, 445 (1986). 